Please note: This paper contains visible codes for electronic publishing. These will not appear in the final version and alignment of tables will be improved.

- Please check that all queries to author [in bold type and square brackets] have been answered, including those in the references and tables. Please do not renumber the references. Any additional references can be added to the bottom of the list and unwanted references deleted from the list without renumbering. The numbering will be corrected automatically during processing

\title{
Should point-of-care ultrasonography replace stethoscopes in acute respiratory failure?
}

Nicholas Smallwood, ${ }^{1}$ consultant in acute internal medicine, Ashley Miller, ${ }^{2}$ consultant in intensive care medicine, Andrew Walden, ${ }^{3}$ consultant in acute internal and intensive care medicine, Mark Hew, consultant in respiratory medicine, ${ }^{4}$ [Q to A Please give one job title for each author, eg researcher, professor, consultant] Tunn Ren Tay, consultant in respiratory and critical care medicine, ${ }^{6}$ Najib M Rahman, professor of respiratory medicine ${ }^{78}$

${ }^{1}$ East Surrey Hospital, Redhill, UK

${ }^{2}$ Royal Shrewsbury Hospital, Shrewsbury, UK

${ }^{3}$ Royal Berkshire Hospital, Reading, UK

${ }^{4}$ Respiratory Medicine, Alfred Hospital, Melbourne, Victoria, Australia

${ }^{5}$ Public Health and Preventive Medicine, Monash University, Melbourne, Victoria, Australia

${ }^{6}$ Department of Respiratory and Critical Care Medicine, Changi General Hospital, Singapore

${ }^{7}$ Oxford Centre for Respiratory Medicine, Churchill Hospital, Oxford, UK

${ }^{8}$ Oxford NIHR Biomedical Research Centre, Oxford, UK

Correspondence to: N Smallwood nicholas.smallwood@nhs.net, M Hew m.hew@alfred.org.au Ultrasonography would be a better diagnostic test than auscultation or chest radiography, say Nicholas Smallwood and colleagues. But Mark Hew and colleagues argue the costs are hard to justify without evidence that it would improve patient outcomes

\section{Yes-Nicholas Smallwood, Ashley Miller, and Andrew Walden}

"The most dangerous phrase in the language is, 'We've always done it this way,", said the US scientist Grace Hopper. And yet, when it comes to diagnosing the cause of acute respiratory failure, the current standard is to use the stethoscope and chest radiography-two technologies with a combined age of over 300 years.

The techniques continue to be used despite their limited diagnostic accuracy mainly because, until recently, there was no available alternative and of the difficulty in abandoning old practices in a traditional profession. 


\section{Current methods not sensitive or specific}

Consider community acquired pneumonia, for example: neither auscultation nor chest radiography are sensitive or specific diagnostic tools. Crackles on auscultation have a sensitivity of $19-67 \%$ and a specificity of 36-96\%, carrying a positive likelihood ratio of 2.3 and a negative likelihood ratio of $0.8 .{ }^{1}$ This limits their use in ruling pneumonia in or out, because their presence or absence can only marginally alter an initial diagnosis.[ok?] Inter-observer reliability is poor, with only $72 \%$ agreement and a kappa value of $0.41 .^{2}$

Chest radiography fares only a little better. Even when reported by radiologists, the presence of infiltrates carries only 59\% agreement, with an overall kappa value of 0.53 (moderate agreement). ${ }^{3}$ When chest radiography is compared with computed tomography to assess for the presence of infiltrates, sensitivity is only $43.5 \%{ }^{4}$

Similar data show the poor diagnostic yield for traditional techniques in the diagnosis of left ventricular dysfunction and other causes of acute respiratory failure. ${ }^{1}$

Conversely, good evidence supports the use of lung ultrasonography in acute respiratory failure. The seminal 2008 single centre study of the bedside lung ultrasound examination protocol $^{5}$ showed an accuracy of $90.5 \%$ in diagnosing the cause of acute respiratory failure in critically ill patients. The methods were standardised, reproducible, and therefore easy to learn and teach.

Since then, international consensus guidelines have highlighted the high quality evidence available. ${ }^{6}$ A recent meta-analysis pooling 12 studies of lung ultrasonography for pneumonia found a sensitivity of $88 \%$, specificity $86 \%$, positive likelihood ratio 5.37 , and negative likelihood ratio 0.13 , indicating its value in ruling in and ruling out pneumonia. ${ }^{7}$ Similar data exist for the diagnosis of pneumothorax, left ventricular failure, and non-cardiogenic pulmonary oedema.

\section{Appropriate use}

We believe the barriers to the implementation of lung ultrasonography can easily be tackled. The capital expenditure costs are substantial. However, machines are becoming cheaper, and increasing availability means training can often occur as part of the patient assessment and review on ward rounds.

Questions also arise around governance: how can we ensure that decision making using lung ultrasound scans is appropriate? We do not audit or review decisions based on auscultation or 
interpretation of radiographs, so to mandate that for every ultrasound examination seems inconsistent. Indeed, a US study spanning 20 years found no lawsuits associated with inappropriate use of bedside ultrasonography ${ }^{8}$; concerns of missing diagnoses and harm are not borne out in practice.

Similarly, it has been suggested that we need studies looking at patient morbidity and mortality before lung ultrasonography is widely used. This is disingenuous. No data exist for chest radiography or computed tomography on these terms because diagnostic tests are assessed against their ability to rule in or rule out conditions accurately. There is no reason to treat ultrasonography differently, and patients have clear benefit in faster, more accurate diagnosis.

Many of the early data, from single centres and for critically ill patients, may not be directly generalisable to internal medicine patients. However, data from emergency department cohorts are showing similar improvements in diagnostic speed and accuracy, ${ }^{9}$ reassuring us that the benefits also exist in an unselected population.

In conclusion, it is no longer appropriate to say, "We've always done it this way." Evidence shows radiography and auscultation are inferior to lung ultrasonography, which has a firm and expanding evidence base. With established training pathways like Core Ultrasound in Intensive Care (CUSIC) and Focused Acute Medicine Ultrasound (FAMUS), ${ }^{10}$ it should be integrated into the assessment of acute respiratory failure.

\section{No-Mark Hew, Tunn Ren Tay, and Najib M Rahman}

A diagnostic test must satisfy key requirements before it can be implemented into clinical practice as the standard of care, given the ensuing legal obligation to provide this care and the corollary of medical negligence if such care is withheld.

Point-of-care ultrasonography for diagnosing acute respiratory failure is problematic for two reasons-efficacy and cost.

\section{Beneficial patient outcomes should be shown}

The efficacy of diagnostic tests extends far beyond diagnostic accuracy. The genuine value of a test lies in its ability to change clinical thinking and management, and ultimately to improve patient outcomes. ${ }^{11}$ Anything short of better patient health is unhelpful. 
Although data show that ultrasongraphy can accurately diagnose the cause of acute respiratory failure in specific situations, there is less evidence for its efficacy in changing management and, as yet, none for it improving patient outcomes ${ }^{\mathrm{x}}$.

The accuracy of ultrasonography is reportedly greater than $90 \%$ for pneumothorax, pneumonia, and pulmonary oedema, but this literature must be interpreted with caution. Many studies were done in highly selected populations, limiting generalisability.

Detection of pneumothorax has been examined only among patients who had experienced trauma, were critically ill, or had undergone procedures with a risk of pneumothorax. ${ }^{11}$ Detection of pulmonary consolidation in acute respiratory failure has been studied only in intensive care. ${ }^{12}$ And pulmonary oedema detection is accurate only when the prevalence of the condition is high. ${ }^{11}$ Sonographic detection of pulmonary embolus is inferior to computed tomography. ${ }^{11}$

Limited data show that point-of-care ultrasound can influence clinical thinking and clinical management. In the emergency department it may shorten time to correct diagnosis, ${ }^{9}$ and on medical wards it can change treatment for acute respiratory failure. ${ }^{13}$ In intensive care units, focused echocardiography, lung, and leg vein ultrasound examinations increased overall diagnostic rates. ${ }^{51415}$ However, all these intensive care studies excluded patients with multiple diagnoses, arguably those for whom diagnostic clarity is needed most. Also, acute respiratory distress syndrome was absent, but in most intensive care cohorts this is the dominant cause of respiratory failure. ${ }^{16}$ On ultrasonography, this syndrome closely mimics pneumonia and pulmonary oedema, and therefore ultrasound examination is likely to have poor discriminatory value when it is prevalent. ${ }^{11}$

The key barrier to implementing point-of-care ultrasonography is the complete absence of any data showing that it improves patient outcomes. The only randomised trial showed faster diagnosis but no other significant benefit. ${ }^{3}$ Consequently, there is an urgent need for randomised trials examining patient outcomes. Because diagnostic tests cannot directly change outcomes, trials must couple test results to prespecified treatments, use standardised treatment protocols, and assess objective patient outcomes. ${ }^{11}$

\section{Cost and potential harm should be measured}

The cost of point-of-care ultrasonography is also unknown. Clearly, there are substantial opportunity costs for equipment and bedside clinician time. A standard of care requires widespread implementation with equitable access wherever acute respiratory failure is 
encountered. Training to a level sufficient to improve (as yet undefined) patient outcomes is needed, requiring sustainable competency programmes and supervisory frameworks to be established. $^{17}$

Finally, the harms of point-of-care ultrasonography must be quantified. The pulmonary artery catheter offers a cautionary tale-implemented with more enthusiasm than evidence, multiple randomised trials subsequently showed no patient benefit but definite documented risks. ${ }^{18}$ Similarly, ultrasonography is not without risk: there is always the possibility (and consequence) of false diagnosis.

\section{Cost-benefit analysis should be performed}

To conclude, current enthusiasm for point-of-care ultrasonography must be channelled into conducting robust controlled studies examining actual patient outcomes in a range of clinical settings. The considerable costs of training, equipment, and potential harms must also be measured. Only if such analyses show overall benefit should it be implemented as standard of care in acute respiratory failure.

Competing interests: All authors have read and understood BMJ policy on declaration of interests and declare the following interests: $\mathrm{MH}$ has received ultrasound course sponsorship in equipment from Fujifilm Sonosite, unrelated to this manuscript. NMR has received technical equipment from Esaote UK for clinical trial work. AW is the director of Box Courses, which delivers point-of-care ultrasonography courses. AM is chair of the CUSIC committee and NS is chair of the FAMUS committee. [Q to A OK? ]

Provenance and peer review: Commissioned; externally peer reviewed. $<$ bok $>1$ Simel DL, Rennie D, Keitz SA. The rational clinical examination: evidence-based clinical diagnosis. McGraw-Hill, 2009. $<$ /bok $>$ $<$ jrn $>2$ Metlay JP, Kapoor WN, Fine MJ. Does this patient have community-acquired pneumonia? Diagnosing pneumonia by history and physical examination. JAMA 1997;278:14405. PubMed doi:10.1001/jama.1997.03550170070035</jrn $>$

$<$ jrn>3 Hopstaken RM, Witbraad T, van Engelshoven JM, Dinant GJ. Inter-observer variation in the interpretation of chest radiographs for pneumonia in community-acquired lower respiratory tract infections. Clin Radiol 2004;59:743-52. PubMed doi:10.1016/i.crad.2004.01.011</jrn> $<$ jrn $>4$ Self WH, Courtney M, McNaughton CD, Wunderink RG, Kline JA. High discordance of chest X-ray and computed tomography for detection of pulmonary opacities in ED patients: implications for diagnosing pneumonia. Am J Emerg Med 2013;31:401-5. PubMed doi:10.1016/j.ajem.2012.08.041</jrn> $<\mathrm{jrn}>5$ Lichtenstein DA, Mezière GA. Relevance of lung ultrasound in the diagnosis of acute respiratory failure: the BLUE protocol. Chest 2008;134:117-25. PubMeddoi:10.1378/chest.07$\underline{2800}</$ jrn $>$ 
$<$ jrn>6 Long L, Zhao HT, Zhang ZY, Wang GY, Zhao HL. Lung ultrasound for the diagnosis of pneumonia in adults: A meta-analysis. Medicine (Baltimore) 2017;96:e5713. PubMed doi:10.1097/MD.0000000000005713 </jrn>

$<$ jrn>7 Volpicelli G, Elbarbary M, Blaivas M, et al; International Liaison Committee on Lung Ultrasound (ILC-LUS) for International Consensus Conference on Lung Ultrasound (ICC-LUS). International evidence-based recommendations for point-of-care lung ultrasound. Intensive Care Med 2012;38:577-91. PubMed doi:10.1007/s00134-012-2513-4</jrn>

$<$ jrn $>8$ Blaivas M, Pawl R. Analysis of lawsuits filed against emergency physicians for point-ofcare emergency ultrasound examination performance and interpretation over a 20 -year period.

Am J Emerg Med 2012;30:338-41. PubMed doi:10.1016/j.ajem.2010.12.016</jrn>

$<$ jrn $>9$ Laursen CB, Sloth E, Lassen AT, et al. Point-of-care ultrasonography in patients admitted with respiratory symptoms: a single-blind, randomised controlled trial. Lancet Respir Med 2014;2:638-46. PubMed doi:10.1016/S2213-2600(14)70135-3</jrn>

$<$ jrn $>10 \quad$ Smallwood N, Dachsel M, Matsa R, Tabiowo E, Walden A. Focused acute medicine ultrasound (FAMUS) - point-of-care ultrasound for the acute medical unit. Acute Med 2016;15:193-6. PubMed $<$ jirn>

$<$ jrn>11 Hew M, Tay TR. The efficacy of bedside chest ultrasound: from accuracy to outcomes. Eur Respir Rev 2016;25:230-46. PubMed doi:10.1183/16000617.0047-2016</jrn>

$<$ jrn $>12$ Hew M, Corcoran JP, Harriss EK, Rahman NM, Mallett S. The diagnostic accuracy of chest ultrasound for CT-detected radiographic consolidation in hospitalised adults with acute respiratory failure: a systematic review. BMJ Open 2015;5:e007838. PubMed doi:10.1136/bmjopen-2015-007838 $<$ jrn $>$

$<$ jrn $>13$ Wallbridge PD, Joosten SA, Hannan LM, et al. A prospective cohort study of thoracic ultrasound in acute respiratory failure: the $C_{3} P O$ protocol. JRSM Open

2017;8:2054270417695055. PubMed doi:10.1177/2054270417695055</jrn>

$<$ jrn $>14$ Silva S, Biendel C, Ruiz J, et al. Usefulness of cardiothoracic chest ultrasound in the management of acute respiratory failure in critical care practice. Chest 2013;144:859-65. PubMed doi:10.1378/chest.13-0167</jrn>

$<$ jrn $>15$ Bataille B, Riu B, Ferre F, et al. Integrated use of bedside lung ultrasound and echocardiography in acute respiratory failure: a prospective observational study in ICU. Chest 2014;146:1586-93. PubMed doi:10.1378/chest.14-0681</jrn>

$<$ jrn $>16$ Bellani G, Laffey JG, Pham T, et al; LUNG SAFE Investigators. ESICM Trials Group. ESICM Trials Group. Epidemiology, patterns of care, and mortality for patients with acute respiratory distress syndrome in intensive care units in 50 countries. JAMA 2016;315:788800. PubMed doi:10.1001/jama.2016.0291</jrn>

$<$ jrn $>17$ Blehar DJ, Barton B, Gaspari RJ. Learning curves in emergency ultrasound education. Acad Emerg Med 2015;22:574-82. PubMed doi:10.1111/acem.12653</jrn>

$<$ jrn $>18$ Marik PE. Obituary: pulmonary artery catheter 1970 to 2013 . Ann Intensive Care 2013;3:38. PubMed doi:10.1186/2110-5820-3-38</jrn $>$

$\mathrm{x}$ Wallbridge P, Steinfort D, Tay TR, Irving L, Hew M. Diagnostic chest ultrasound for acute respiratory failure. Respir Med. 2018 Aug;141:26-36. doi: 10.1016/j.rmed.2018.06.018. 